Elect. Comm. in Probab. 14 (2009), 202-209

ELECTRONIC

COMMUNICATIONS

in PROBABILITY

\title{
AN OPTIMAL ITÔ FORMULA FOR LÉVY PROCESSES
}

NATHALIE EISENBAUM

LPMA, UMR 7599, CNRS-Université Paris 6 - 4, place Jussieu, 75252 Paris Cedex 05, France

email: nathalie.eisenbaum@upmc.fr

ALEXANDER WALSH

LPMA, UMR 7599, CNRS-Université Paris 6 - 4, place Jussieu, 75252 Paris Cedex 05, France

email: awalshz@gmail.com

Submitted December 15, 2008, accepted in final form April 20, 2009

AMS 2000 Subject classification: 60G44, 60H05, 60J55, $60 \mathrm{~J} 65$

Keywords: stochastic calculus, Lévy process, local time, Itô formula.

Abstract

Several Itô formulas have been already established for Lévy processes. We explain according to which criteria they are not optimal and establish an extended Itô formula that satisfies that criteria. The interest, in particular, of this formula, is to obtain the explicit decomposition of $F\left(X_{t}, t\right)$, for $X$ Lévy process and $F$ deterministic function with locally bounded first order Radon-Nikodym derivatives, as the sum of a Dirichlet process and a bounded variation process.

\section{Introduction and main results}

Let $X$ be a general real-valued Lévy process with characteristic triplet $(a, \sigma, v)$, i.e. its characteristic exponent is equal to

$$
\psi(u)=i u a-\sigma^{2} \frac{u^{2}}{2}+\int_{\mathbb{R}}\left(e^{i u y}-1-i u y 1_{\{|y| \leq 1\}}\right) v(d y)
$$

where $a$ and $\sigma$ are real numbers and $v$ is a Lévy measure. We will denote by $\left(\sigma B_{t}, t \geq 0\right)$ the Brownian component of $X$. Let $F$ be a $C^{2,1}$ function from $\mathbb{R} \times \mathbb{R}^{+}$to $\mathbb{R}$. The classical Itô formula gives

$$
\begin{aligned}
F\left(X_{t}, t\right) & =F\left(X_{0}, 0\right)+\int_{0}^{t} \frac{\partial F}{\partial t}\left(X_{s-}, s\right) d s \\
& +\int_{0}^{t} \frac{\partial F}{\partial x}\left(X_{s-}, s\right) d X_{s}+\frac{\sigma^{2}}{2} \int_{0}^{t} \frac{\partial^{2} F}{\partial x^{2}}\left(X_{s}, s\right) d s \\
& +\sum_{0<s \leq t}\left\{F\left(X_{s}, s\right)-F\left(X_{s-}, s\right)-\frac{\partial F}{\partial x}\left(X_{s-}, s\right) \Delta X_{s}\right\}
\end{aligned}
$$


This formula can be rewritten under the following form (see [8]): $\left(F\left(X_{t}, t\right), t \geq 0\right)$ is a semimartingale admitting the decomposition

$$
F\left(X_{t}, t\right)=F\left(X_{0}, 0\right)+M_{t}+V_{t}
$$

where the local martingale $M$ and the adapted with bounded variation process $V$ are given by

$$
\begin{gathered}
M_{t}=\sigma \int_{0}^{t} \frac{\partial F}{\partial x}\left(X_{s-}, s\right) d B_{s}+\int_{0}^{t} \int_{\{|y|<1\}}\left\{F\left(X_{s-}+y, s\right)-F\left(X_{s-}, s\right)\right\} \tilde{\mu}_{X}(d y, d s) \\
V_{t}=\sum_{0<s \leq t}\left\{F\left(X_{s}, s\right)-F\left(X_{s-}, s\right)\right\} 1_{\left\{\left|\Delta X_{s}\right| \geq 1\right\}}+\int_{0}^{t} \mathscr{A} F\left(X_{s}, s\right) d s
\end{gathered}
$$

where $\tilde{\mu}_{X}(d y, d s)$ denotes the compensated Poisson measure associated to the jumps of $X$, and $\mathscr{A}$ is the operator associated to $X$ defined by

$$
\begin{aligned}
\mathscr{A} G(x, t)= & \frac{\partial G}{\partial t}(x, t)+a \frac{\partial G}{\partial x}(x, s)+\frac{1}{2} \sigma^{2} \frac{\partial^{2} G}{\partial x^{2}}(x, t) \\
& +\int_{\mathbb{R}}\left\{G(x+y, t)-G(x, t)-y \frac{\partial G}{\partial x}(x, t)\right\} 1_{(|y|<1)} v(d y)
\end{aligned}
$$

for any function $G$ defined on $\mathbb{R} \times \mathbb{R}^{+}$, such that $\frac{\partial G}{\partial x}, \frac{\partial G}{\partial t}$ and $\frac{\partial^{2} G}{\partial x^{2}}$ exist as Radon-Nikodym derivatives with respect to the Lebesgue measure and the integral is well defined. The later condition is satisfied when $\frac{\partial^{2} G}{\partial x^{2}}$ is locally bounded.

Note that the existence of locally bounded first order Radon-Nikodym derivatives alone guarantees the existence of

$$
F\left(X_{t}, t\right)-F\left(X_{0}, 0\right)-\int_{0}^{t} \frac{\partial F}{\partial t}\left(X_{s-}, s\right) d s-\int_{0}^{t} \frac{\partial F}{\partial x}\left(X_{s-}, s\right) d X_{s}
$$

but then to say that this expression coincides with

$$
\frac{\sigma^{2}}{2} \int_{0}^{t} \frac{\partial^{2} F}{\partial x^{2}}\left(X_{s}, s\right) d s+\sum_{0<s \leq t}\left\{F\left(X_{s}, s\right)-F\left(X_{s-}, s\right)-\frac{\partial F}{\partial x}\left(X_{s-}, s\right) \Delta X_{s}\right\}
$$

we need to assume much more on $F$.

In that sense one might say that the classical Itô formula is not optimal. The interest of an optimal formula is two-fold. It allows to expand $F\left(X_{t}, t\right)$ under minimal conditions on $F$ but also to know explicitly the structure of the process $F\left(X_{t}, t\right)$. Such an optimal formula has been established in the particular case when $X$ is a Brownian motion [4]. Indeed in that case, under the minimal assumption on $F$ for the existence of (1.5), namely that $F$ admits locally bounded first order Radon-Nikodym derivatives, we know that this expression coincides with

$$
-\frac{1}{2} \int_{0}^{t} \int_{\mathbb{R}} \frac{\partial F}{\partial x}(x, s) d L_{s}^{x}
$$

where $\left(L_{s}^{x}, x \in \mathbb{R}, s \geq 0\right)$ is the local time process of $X$. Moreover the process $\left(\int_{0}^{t} \int_{\mathbb{R}} \frac{\partial F}{\partial x}(x, s) d L_{s}^{x}, t \geq 0\right)$ has a 0-quadratic energy. 
In the general case, various extensions of (1.1) have been established. We will quote here only the extensions exploiting the notion of local times, we send to [4] for a more exhaustive bibliography. Meyer [9] has been the first to relax the assumption on $F$ by introducing an integral with respect to local time, followed then by Bouleau and Yor [3], Azéma et al [1], Eisenbaum [4], [5], Ghomrasni and Peskir [7], Eisenbaum and Kyprianou [6]. In the discontinuous case, none of the obtained Itô formulas is optimal because of the presence of the expression $\sum_{0<s \leq t}\left\{F\left(X_{s}, s\right)-F\left(X_{s-}, s\right)-\right.$ $\left.\frac{\partial F}{\partial x}\left(X_{s-}, s\right) \Delta X_{s}\right\}$.

The Itô formula for Lévy processes presented below in Theorem 1.1 , is available for $X$ admitting a Brownian component. It lightens the condition on the jumps of $X$ required by [5], and it also lightens the condition on the first order derivatives of $F$ required by [6]. Besides it is optimal. To introduce it we need the operator $I$ defined on the set of locally bounded measurable functions $G$ on $\mathbb{R} \times \mathbb{R}^{+}$by

$$
I G(x, t)=\int_{0}^{x} G(y, t) d y .
$$

We will denote the Markov local time process of $X$ by $\left(L_{t}^{x}, x \in \mathbb{R}, t \geq 0\right)$.

Theorem 1.1. : Assume that $\sigma \neq 0$. Let $F$ be a function from $\mathbb{R} \times \mathbb{R}^{+}$to $\mathbb{R}$ such that $\frac{\partial F}{\partial x}$ and $\frac{\partial F}{\partial t}$ exist as Radon-Nikodym derivatives with respect to the Lebesgue measure and are locally bounded. Then the process $\left(F\left(X_{t}, t\right), t \geq 0\right)$ admits the following decomposition

$$
F\left(X_{t}, t\right)=F\left(X_{0}, 0\right)+M_{t}+V_{t}+Q_{t}
$$

with $M$ the local martingale given by (1.3), $V$ is the bounded variation process

$$
V_{t}=\sum_{0 \leq s \leq t}\left\{F\left(X_{s}, s\right)-F\left(X_{s-}, s\right)\right\} 1_{\left\{\left|\Delta X_{s}\right| \geq 1\right\}}
$$

and $Q$ the following adapted process with 0-quadratic variation

$$
Q_{t}=-\int_{0}^{t} \int_{\mathbb{R}} \mathscr{A} I F(x, s) d L_{s}^{x} .
$$

As a simple application of Theorem 1.1 consider the example of the function $F(x, s)=|x|$ in the case $\int_{0}^{1} y v(d y)=+\infty$. This function does not satisfy the assumption of Theorem 3 of [6] nor $X$ does satisfy the assumption of Theorem 2.2 in [5]. But, thanks to Theorem 1.1, we immediately obtain Tanaka's formula.

The proofs are presented in Section 2.

\section{Proofs}

We first remind the meaning of integration with respect to the semimartingale local time process of $X$ denoted $\left(\ell_{s}^{x}, x \in \mathbb{R}, s \geq 0\right)$. Theorem 1.1 is expressed in terms of the Markov local time process $\left(L_{s}^{x}, x \in \mathbb{R}, s \geq 0\right)$. The two processes are connected by:

$\left(L_{s}^{x}, x \in \mathbb{R}, s \geq 0\right)=\left(\frac{1}{\sigma^{2}} \ell_{s}^{x}, x \in \mathbb{R}, s \geq 0\right)$.

Let $\sigma B$ be the Brownian component of $X$. Defined the norm $\|$.$\| of a measurable function f$ from $\mathbb{R} \times \mathbb{R}_{+}$to $\mathbb{R}$ by

$$
\|f\|=2 \mathbb{E}\left(\int_{0}^{1} f^{2}\left(X_{s}, s\right) d s\right)^{1 / 2}+\mathbb{E}\left(\int_{0}^{1}\left|f\left(X_{s}, s\right)\right| \frac{\left|B_{s}\right|}{s} d s\right) .
$$


In [6], integration with respect to $\ell$ of locally bounded mesurable function $f$ has been defined by

$$
\int_{0}^{t} \int_{\mathbb{R}} f(x, s) d \ell_{s}^{x}=\sigma \int_{0}^{t} f\left(X_{s-}, s\right) d B_{s}+\sigma \int_{1-t}^{1} f\left(\hat{X}_{s-}, 1-s\right) d \hat{B}_{s}, \quad 0 \leq t \leq 1
$$

where $\hat{B}$ and $\hat{X}$ are the time reversal at 1 of $B$ and $X$.

We have the following properties:

(i) $\mathbb{E}\left(\left|\int_{0}^{t} \int_{\mathbb{R}} f(x, s) d \ell_{s}^{x}\right|\right) \leq|\sigma||| f||$.

(ii) If $f$ admits a locally bounded Radon-Nikodym derivative with respect to $x$, then: $\int_{0}^{t} \int_{\mathbb{R}} f(x, s) d \ell_{s}^{x}=$ $-\sigma^{2} \int_{0}^{t} \frac{\partial f}{\partial x}\left(X_{s}, s\right) d s$.

(iii) The process $\left(\int_{0}^{t} \int_{\mathbb{R}} f(x, s) d \ell_{s}^{x}, 0 \leq t \leq 1\right)$ has 0 -quadratic variation.

Proof of Theorem 1.1: We start by assuming that $F$ and $\frac{\partial F}{\partial x}$ are bounded. We set

$$
F_{n}(x, t)=\iint_{\mathbb{R}^{2}} F(x-y / n, t-s / n) f(y) h(s) d y d s
$$

where $f$ and $h$ are nonnegative $C^{\infty}$ functions with compact supports such that : $\int_{\mathbb{R}} f(x) d x=$ $\int_{\mathbb{R}} h(x) d x=1$. Thanks to the usual Itô formula we have:

$$
\begin{aligned}
F_{n}\left(X_{t}, t\right)=\quad & F_{n}(0,0)+\sigma \int_{0}^{t} \frac{\partial F_{n}}{\partial x}\left(X_{s-}, s\right) d B_{s}+\int_{0}^{t} \frac{\partial F_{n}}{\partial t}\left(X_{s}, s\right) d s \\
& +a \int_{0}^{t} \frac{\partial F_{n}}{\partial x}\left(X_{s}, s\right) d s+\sum_{0 \leq s \leq t}\left\{F_{n}\left(X_{s}, s\right)-F_{n}\left(X_{s-}, s\right)\right\} 1_{\left\{\left|\Delta X_{s}\right| \geq 1\right\}} \\
& +\int_{0}^{t} \int_{\mathbb{R}}^{t}\left\{F_{n}\left(X_{s-}+y, s\right)-F_{n}\left(X_{s-}, s\right)\right\} 1_{\{|y|<1\}} \tilde{\mu}(d s, d y) \\
& +\frac{\sigma^{2}}{2} \int_{0}^{t} \frac{\partial^{2} F_{n}}{\partial x^{2}}\left(X_{s}, s\right) d s \\
& +\int_{0}^{t} \int_{-1}^{1}\left\{F_{n}\left(X_{s}+y, s\right)-F_{n}\left(X_{s}, s\right)-\frac{\partial F_{n}}{\partial x}\left(X_{s}, s\right) y\right\} v(d y) d s
\end{aligned}
$$

With the same arguments as in the proof of Theorem 2.2 of [5], we see that as $n$ tends to $\infty$, $F_{n}\left(X_{t}, t\right)$ and each of the first five terms of the RHS of (2.2) converges at least in probability to the corresponding expression with $F$ replacing $F_{n}$. Besides we note that

$$
\begin{aligned}
\int_{0}^{t} \frac{\partial F}{\partial t}\left(X_{s}, s\right) d s & =-\frac{1}{\sigma^{2}} \int_{0}^{t} \int_{\mathbb{R}}\left(\int_{0}^{x} \frac{\partial F}{\partial t}(y, s) d y\right) d \ell_{s}^{x} \\
& =-\int_{0}^{t} \int_{\mathbb{R}}\left(\frac{\partial}{\partial t} \int_{0}^{x} F(y, s) d y\right) d L_{s}^{x}
\end{aligned}
$$

since $\frac{\partial F}{\partial t}$ is locally bounded. Hence we have:

$$
\int_{0}^{t} \frac{\partial F}{\partial t}\left(X_{s}, s\right) d s=-\int_{0}^{t} \int_{\mathbb{R}} \frac{\partial(I F)}{\partial t}(x, s) d L_{s}^{x} .
$$


Since : $F(x, s)=\frac{\partial(I F)}{\partial x}(x, s)$, we immediately obtain:

$$
a \int_{0}^{t} \frac{\partial F}{\partial x}\left(X_{s}, s\right) d s=-\int_{0}^{t} \int_{\mathbb{R}} a \frac{\partial(I F)}{\partial x}(x, s) d L_{s}^{x} .
$$

The convergence in $L^{2}$ of the sixth term of the RHS is obtained with the same proof as in [6]. The limit is equal to

$$
\int_{0}^{t} \int_{\mathbb{R}}\left\{F\left(X_{s-}+y, s\right)-F\left(X_{s-}, s\right)\right\} 1_{\{|y|<1\}} \tilde{u}(d s, d y)
$$

For the seventh term of the RHS of (2.2), we note that :

$\frac{\sigma^{2}}{2} \int_{0}^{t} \frac{\partial^{2} F_{n}}{\partial x^{2}}\left(X_{s}, s\right) d s=-\frac{1}{2} \int_{0}^{t} \int_{\mathbb{R}} \frac{\partial F_{n}}{\partial x}(x, s) d \ell_{s}^{x}$. Thanks to the properties (i) and (ii) of the integration with respect to the local times, this expression converges in $L^{1}$ to $-\frac{1}{2} \int_{0}^{t} \int_{\mathbb{R}} \frac{\partial F}{\partial x}(x, s) d \ell_{s}^{x}$. We can obviously write:

$$
-\frac{1}{2} \int_{0}^{t} \int_{\mathbb{R}} \frac{\partial F}{\partial x}(x, s) d \ell_{s}^{x}=-\frac{\sigma^{2}}{2} \int_{0}^{t} \int_{\mathbb{R}} \frac{\partial^{2}(I F)}{\partial x^{2}}(x, s) d L_{s}^{x} .
$$

We now study the convergence of the last term of the RHS of (2.2). We have:

$$
\begin{aligned}
\int_{0}^{t} \int_{-1}^{1} & \left\{F_{n}\left(X_{s}+y, s\right)-F_{n}\left(X_{s}, s\right)-\frac{\partial F_{n}}{\partial x}\left(X_{s}, s\right) y\right\} v(d y) d s \\
= & -\int_{0}^{t} \int_{\mathbb{R}} H_{n}(x, s) d L_{s}^{x}
\end{aligned}
$$

where: $H_{n}(x, s)=\int_{0}^{x} \int_{-1}^{1}\left\{F_{n}(z+y, s)-F_{n}(z, s)-\frac{\partial F_{n}}{\partial x}(z, s) y\right\} v(d y) d z$. We have:

$$
\begin{aligned}
\mid F_{n}(z+y, s) & -F_{n}(z, s)-\frac{\partial F_{n}}{\partial x}(z, s) y \mid 1_{\{|y|<1\}} \\
& =\left|\int_{z}^{z+y} \frac{\partial F_{n}}{\partial x}(v, t)-\frac{\partial F_{n}}{\partial x}(z, t) d v\right| 1_{\{|y|<1\}} \\
& \leq y^{2} \sup \left|\frac{\partial^{2} F_{n}}{\partial x^{2}}\right| 1_{\{|y|<1\}} .
\end{aligned}
$$

Noting that: $\frac{\partial^{2} F_{n}}{\partial x^{2}}(x, t)=n^{2} \iint_{\mathbb{R}^{2}} F(x-y / n, t-s / n) f^{\prime \prime}(y) h(s) d y d s$, we obtain $\mid F_{n}(z+y, s)-$ $F_{n}(z, s)-\frac{\partial F_{n}}{\partial x}(z, s) y\left|1_{\{|y|<1\}} \leq \operatorname{csten}^{2} y^{2} 1_{\{|y|<1\}} \sup \right| F \mid$

Consequently :

$$
\begin{aligned}
H_{n}(x, s) & =\int_{-1}^{1} \int_{0}^{x}\left\{F_{n}(z+y, s)-F_{n}(z, s)-\frac{\partial F_{n}}{\partial x}(z, s) y\right\} d z v(d y) \\
= & \int_{-1}^{1}\left\{\int_{0}^{x+y} F_{n}(z, s) d z-\int_{0}^{x} F_{n}(z, s) d z-y F_{n}(x, s)+y F_{n}(0, s)-\int_{0}^{y} F_{n}(z, s) d z\right\} v(d y) \\
= & G_{n}(x, s)+\int_{-1}^{1}\left(y F_{n}(0, s)-\int_{0}^{y} F_{n}(z, s) d z\right) v(d y)
\end{aligned}
$$


where $G_{n}(x, s)=\int_{-1}^{1}\left(I F_{n}(x+y, s)-I F_{n}(x, s)-y F_{n}(x, s)\right) v(d y)$. Thanks to Corollary 8 of [6], we know that

$$
\int_{0}^{t} \int_{\mathbb{R}} H_{n}(x, s) d L_{s}^{x}=\int_{0}^{t} \int_{\mathbb{R}} G_{n}(x, s) d L_{s}^{x} .
$$

By dominated convergence, we have as $n$ tends to $\infty$ for every $(x, s)$

$$
I F_{n}(x+y, s)-I F_{n}(x, s)-y F_{n}(x, s) \rightarrow I F(x+y, s)-I F(x, s)-y F(x, s) .
$$

Besides, for every $n:\left|I F_{n}(x+y, s)-I F_{n}(x, s)-y F_{n}(x, s)\right| \leq y^{2} 1_{\{|y|<1\}}$ sup $\left|\frac{\partial F}{\partial x}\right|$, hence for every $(x, s): G_{n}(x, s)$ tends to $G(x, s)$, where

$$
G(x, s)=\int_{\mathbb{R}}(I F(x+y, s)-I F(x, s)-y F(x, s)) 1_{\{|y|<1\}} v(d y) .
$$

By dominated convergence, $\left(G_{n}\right)_{n>0}$ converges for the norm $\|$.$\| to G$. Consequently the limit of the last term of the RHS of (2.2) is equal by (2.7) and (2.8) to

$$
-\int_{0}^{t} \int_{\mathbb{R}} \int_{\mathbb{R}}(I F(x+y, s)-I F(x, s)-y F(x, s)) 1_{\{|y|<1\}} v(d y) d L_{s}^{x} .
$$

Summing all the limits (2.3), (2.4), (2.5), (2.6) and (2.9), we finally obtain

$$
\begin{aligned}
F\left(X_{t}, t\right) & =F\left(X_{0}, 0\right)+\sigma \int_{0}^{t} \frac{\partial F}{\partial x}\left(X_{s-}, s\right) d B_{s} \\
& +\int_{0}^{t} \int_{\mathbb{R}}\left\{F\left(X_{s-}+y, s\right)-F\left(X_{s-}, s\right)\right\} 1_{\{|y|<1 \mid\}} \tilde{\mu}(d s, d y) \\
& +\sum_{0<s \leq t}^{t}\left\{F\left(X_{s}, s\right)-F\left(X_{s-}, s\right)\right\} 1_{\left\{\left|\Delta X_{s}\right| \geq 1\right\}} \\
& -\int_{0}^{t} \int_{\mathbb{R}}\left\{\frac{\partial(I F)}{\partial t}(x, s)+a \frac{\partial(I F)}{\partial x}(x, s)+\frac{\sigma^{2}}{2} \frac{\partial^{2}(I F)}{\partial x^{2}}(x, s)\right\} d L_{s}^{x} \\
& -\int_{0}^{t} \int_{\mathbb{R}}\left\{\int\{I F(x+y, s)-I F(x, s)-y F(x, s)\} 1_{\{|y|<1\}} v(d y)\right\} d L_{s}^{x} .
\end{aligned}
$$

which summarizes in

$$
\begin{aligned}
F\left(X_{t}, t\right)= & F\left(X_{0}, 0\right)+\sigma \int_{0}^{t} \frac{\partial F}{\partial x}\left(X_{s-}, s\right) d B_{s} \\
& +\int_{0}^{t} \int_{\mathbb{R}}\left\{F\left(X_{s-}+y, s\right)-F\left(X_{s-}, s\right)\right\} 1_{\{|y|<1\}} \tilde{\mu}(d s, d y) \\
& +\sum_{0<s \leq t}\left\{F\left(X_{s}, s\right)-F\left(X_{s-}, s\right)\right\} 1_{\left\{\left|\Delta X_{s}\right| \geq 1\right\}}-\int_{0}^{t} \int_{\mathbb{R}} \mathscr{A} I F(x, s) d L_{s}^{x} .
\end{aligned}
$$

In the general case, we set:

$$
\tilde{F}_{n}(x, s)=F(x, s) 1_{\left[a_{n}, b_{n}\right]}(x)+F\left(a_{n}, s\right) 1_{\left(-\infty, a_{n}\right)}(x)+F\left(b_{n}, s\right) 1_{\left(b_{n}, \infty\right)}(x)
$$


where $\left(-a_{n}\right)_{n \in \mathbb{N}}$ and $\left(b_{n}\right)_{n \in \mathbb{N}}$ are two positive real sequences increasing to $\infty$.

We write $(2.10)$ for $\tilde{F}_{n}$ and stop the process $\left(\tilde{F}_{n}\left(X_{s}, s\right), 0 \leq s \leq 1\right)$ at $T_{m}=1 \wedge \inf \left\{s \geq 0:\left|X_{s}\right|>\right.$ $m$ \}. We let $n$ tend to $\infty$ and then $m$ tend to $\infty$. The behavior of two terms deserves specific explanations, the other terms converging respectively to the expected expressions.

The first one is : $\int_{0}^{t \wedge T_{m}} \int_{\mathbb{R}}\left\{\int\left\{I \tilde{F}_{n}(x+y, s)-I \tilde{F}_{n}(x, s)-y \tilde{F}_{n}(x, s)\right\} 1_{\{|y|<1\}} v(d y)\right\} d L_{s}^{x}$. Thanks to the definition of the integral with respect to local time (2.1), it is equal to

$$
\frac{1}{\sigma} \int_{0}^{t \wedge T_{m}} \tilde{H}_{n}\left(X_{s-}, s\right) d B_{s}+\frac{1}{\sigma} \int_{1-\left(t \wedge T_{m}\right)}^{1} \tilde{H}_{n}\left(\hat{X}_{s-}, s\right) d \hat{B}_{s}
$$

where $\tilde{H}_{n}(x, s)=\int\left\{I \tilde{F}_{n}(x+y, s)-I \tilde{F}_{n}(x, s)-y \tilde{F}_{n}(x, s)\right\} 1_{\{|y|<1\}} v(d y)$.

We set $H(x, s)=\int\{I F(x+y, s)-I F(x, s)-y F(x, s)\} 1_{\{|y|<1\}} v(d y)$.

We can choose $n$ big enough to have $\left|a_{n}\right|$ and $b_{n}$ bigger than $m+1$. Hence (2.11) is equal to

$$
\frac{1}{\sigma} \int_{0}^{t \wedge T_{m}} H\left(X_{s-}, s\right) d B_{s}+\frac{1}{\sigma} \int_{1-\left(t \wedge T_{m}\right)}^{1} H\left(\hat{X}_{s-}, s\right) d \hat{B}_{s} .
$$

For every $\epsilon>0$

$$
\begin{aligned}
\mathbb{P}\left(\sup _{0 \leq t \leq 1} \mid \int_{1-\left(t \wedge T_{m}\right)}^{1} H\left(\hat{X}_{s-}, s\right) d \hat{B}_{s}\right. & \left.-\int_{1-t}^{1} H\left(\hat{X}_{s-}, s\right) d \hat{B}_{s} \mid \geq \epsilon\right) \\
& \leq \mathbb{P}\left(T_{m}<1\right) \\
& =\mathbb{P}\left(\sup _{0 \leq t \leq 1}\left|X_{t}\right|>m\right)
\end{aligned}
$$

which shows that as $m$ tends to $\infty, \int_{1-\left(t \wedge T_{m}\right)}^{1} H\left(\hat{X}_{s-}, s\right) d \hat{B}_{s}$ converges in probability uniformly on $[0,1]$ to $\int_{1-t}^{1} H\left(\hat{X}_{s-}, s\right) d \hat{B}_{s}$. Similarly $\int_{0}^{t \wedge T_{m}} H\left(X_{s-}, s\right) d B_{s}$ converges in probability to $\int_{0}^{t} H\left(X_{s-}, s\right) d B_{s}$. Consequently as $m$ tends to $\infty$, (2.11) converges to

$$
\int_{0}^{t} \int_{\mathbb{R}}\left\{\int\{I F(x+y, s)-I F(x, s)-y F(x, s)\} 1_{\{|y|<1\}} v(d y)\right\} d L_{s}^{x} .
$$

The second term is : $\int_{0}^{t} \int_{\mathbb{R}}\left\{\tilde{F}_{n}\left(X_{s-}+y, s\right)-\tilde{F}_{n}\left(X_{s-}, s\right)\right\} 1_{\left\{s<T_{m}\right\}} 1_{\{|y|<1 \mid\}} \tilde{\mu}(d s, d y)$. For $n$ big enough such that $\left|a_{n}\right|, b_{n}>m$, this term is equal to

$\int_{0}^{t} \int_{\mathbb{R}}\left\{F\left(X_{s-}+y, s\right)-F\left(X_{s-}, s\right)\right\} 1_{\left\{s<T_{m}\right\}} 1_{\{|y|<1 \mid\}} \tilde{\mu}(d s, d y)$. As Ikeda and Watanabe [8], we then denote by $\left(\int_{0}^{t} \int_{\mathbb{R}}\left\{F\left(X_{s-}+y, s\right)-F\left(X_{s-}, s\right)\right\} 1_{\{|y|<1 \mid\}} \tilde{\mu}(d s, d y), 0 \leq t \leq 1\right)$ the local martingale $\left(Y_{t}, 0 \leq t \leq 1\right)$ defined by :

$$
Y_{t \wedge T_{m}}=\int_{0}^{t} \int_{\mathbb{R}}\left\{\tilde{F}\left(X_{s-}+y, s\right)-\tilde{F}\left(X_{s-}, s\right)\right\} 1_{\left\{s<T_{m}\right\}} 1_{\{|y|<1\}} \tilde{\mu}(d s, d y) .
$$




\section{References}

[1] Azéma J., Jeulin T., Knight F., Yor M. : Quelques calculs de compensateurs impliquant l'injectivité de certains processus croissants. Séminaire de Probabilités XXXII LNM 1686, 316-327 (1998). MR1655302

[2] Bertoin J. : Lévy processes. Cambridge University Press. (1996). MR1406564

[3] Bouleau N., Yor M. : Sur la variation quadratique des temps locaux de certaines semimartingales .C.R.Acad. Sc. Paris 292, 491-494 (1981). MR0612544

[4] Eisenbaum N. : Integration with respect to local time. Potential Analysis 13, 303-328 (2000). MR1804175

[5] Eisenbaum N. : Local time-space stochastic calculus for Lévy processes.Stoch.Proc. and Appl.,116, 5 , 757-778 (2006). MR2218334

[6] Eisenbaum N. and Kyprianou A. : On the parabolic generator of a general one-dimensional Lévy process.Electron. Commun. Probab. 13,198-209 (2008). MR2399282

[7] Ghomrasni R., Peskir G. : Local time-space calculus and extensions of Itô's formula. Proc. High Dim. Probab. III (Sandbjerg 2002) Prog.Probab. 55, Birkhäuser Basel, 177-192 (2003). MR2033888

[8] Ikeda N. and Watanabe S. : Stochastic differential equations and diffusion processes. Second edition. North-Holland Mathematical Library (1989). MR1011252

[9] Meyer P.A. : Un cours sur les intégrales stochastiques. Séminaire de Probabilités $X$ - Lect. Notes 511 246-354 (1976). MR0501332 